\title{
Research on the Relation between Household Income and Financial Asset Allocation-Take Beijing-Tianjin-Hebei Region as an Example
}

\author{
Yuhan Sun ${ }^{1, \text { a, * }}$ \\ ${ }^{1}$ School of Economics, Hebei University, Baoding 017000, China \\ axe7997@163.com
}

Keywords: Beijing-Tianjin-Hebei Region, Income Gap, Financial Asset Allocation

\begin{abstract}
Imbalance of economic development in Beijing-Tianjin-Hebei Region and the widening of household income gap of residents have seriously restricted the process of the coordinated development between Beijing-Tianjin-Hebei Region. However, the government made more use of macroscopic means and ignored the microcosmic effect of family. The work utilizes stata software to conduct a data analysis to demonstrate the relationship between the two by using relevant data of Household Income and Asset Allocation in 2013 which was published by China Household Finance Survey and Research Center (CHFS) of South western University of Finance and Economics. The research conclusions are that the total amount of household income and total amount of financial asset have a positive correlation relation and the increase in household income is affected by structure of financial asset allocation. In order to solve the above problems, this paper offers the suggestions, including increasing the amount and the type of financial investments in Hebei region and improving the availability of financial assets of Hebei residents; And for Beijing and Tianjin region with higher income, mainly adjusting the structure of investment-oriented financial asset allocation and trying the financial asset allocation modes except for house property, stock and deposit account. This has very important practical significance to improve the wealth level of residents in the Beijing-Tianjin-Hebei region and to optimize the financial asset allocation.
\end{abstract}

\section{Introduction}

General Secretary $\mathrm{Xi}$ Jinping defines the development goals in Outline of Beijing-Tianjin-Hebei Coordinated Development Plan as: we need to deepen reform, drive innovation and set demonstration pilots. Pushing the coordinated development orderly shall achieve remarkable results by 2017. From medium term to 2020, we shall achieve positive results on co-construction and sharing of public service; The coordinated development mechanism shall operate effectively and the regional development gaps tend to be narrow; A new situation of coordinated development and mutual benefit in Beijing-Tiajin-Hebei region shall take initial shape. 


\section{Organization of the Text}

\subsection{The Gap of Household Income presents the Trend of Expansion}

Table 1 Statement of Annual Income of Residents in Beijing-Tianjin-Hebei Region in 2013

Unit: Yuan

\begin{tabular}{lccc}
\hline & Total Income & Mean Value & Median \\
\hline Beijing & 81705572 & 106665 & 65900 \\
Tianjin & 38242402 & 69785 & 50000 \\
Hebei & 5125210 & 93185 & 84000 \\
\hline
\end{tabular}

DATA Source: China Household Finance Survey and Research Center (CHFS)2013

According to survey data of household incomes in three regions of Beijing, Tianjin and Hebei in 2013, we can basically see the following two problems: Firstly, the gap of total amount of incomes in three regions of Beijing, Tianjin and Hebei is obvious and the total amount of incomes in Hebei province has a big gap with the total amount of income in Beijing and Tianjin. Secondly, see from the gap of mean value and median in three regions, gaps of the internal income also exist between Beijing, Tianjin and Hebei.

\subsection{The Status of Financial Asset Allocation will Affect the Level of Household Income}

Table 2 Average Annual Household Income in Beijing, Tianjin and Hebei Region in 2013

Unit: Yuan

\begin{tabular}{cccc}
\hline & Beijing & Tianjin & Hebei \\
\hline Family with Financial Allocation & 91922.41 & 60393.15 & 39297.73 \\
Family With non-financial Allocation & 54930.43 & 38159.06 & 36053.33 \\
\hline
\end{tabular}

DATA Source: China Household Finance Survey and Research Center (CHFS)2013

Table 2 indicates that the financial asset allocations made by the residents in Beijing and Tianjin can effectively raise their household incomes, but the earnings gained from asset allocations made by some families in Hebei are poor and then it further indicates that there is something wrong in the methods of financial assets allocations made by the families in Hebei region and the availability of Hebei residents on financial products is not too high.

\section{Case Demonstration Part}

In the aspect of household financial asset allocation, we have selected: Total amount of Financial Asset(finsum), Earnings gained from Financial Asset(fininter), Proportion of Current Deposit(cdep), Proportion of Fixed Term Deposit(tdep), Proportion of Stock Value(stock), Proportion of Face Value of Bond(bond), Proportion of Face Value of Fund(fund) and Proportion of Bank Financial Products(bankfin).

Research Method: Utilize stata software to make a cross-section data analysis. And in consideration that four of them have the common investment attribute, merge them to form a new variable, that is the proportion of investment-oriented financial product(suminv)

We conduct the categorical regression in accordance with different income levels and in combination with distribution of samples' income levels, they are divided into three sections, [1300,50000] , [50000,150000] , [15000,548100] which respectively represent the low, middle and high income class. 
Table 3 Regression Results of Annual Income in 2013

\begin{tabular}{lccc}
\hline VARIABLES & $\mathrm{m} 5$ & $\mathrm{~m} 6$ & $\mathrm{~m} 7$ \\
lnincome & lnincome & lnincome \\
\hline \multirow{2}{*}{ lnfinsum } & $0.149^{* * *}$ & 0.00779 & 0.0330 \\
& $(0.0270)$ & $(0.0238)$ & $(0.0488)$ \\
cdep & $0.244^{* *}$ & 0.0934 & 0.124 \\
& $(0.116)$ & $(0.138)$ & $(0.229)$ \\
suminv & $0.492^{* * *}$ & 0.0565 & 0.0407 \\
& $(0.124)$ & $(0.138)$ & $(0.267)$ \\
Constant & $9.341^{* * *}$ & $10.78^{* * *}$ & $9.808 * * *$ \\
& $(0.329)$ & $(0.285)$ & $(0.575)$ \\
Observations & 599 & 361 & 141 \\
R-squared & 0.116 & 0.001 & 0.004 \\
\hline
\end{tabular}

Robust standard errors in parentheses

$* * * \mathrm{p}<0.01, * * \mathrm{p}<0.05, * \mathrm{p}<0.1$

\section{Conclusion}

See from Regression Results of the Above Table, model 8 is the low income family and both of total amount and structure of financial asset have no effect on the household income; Model 9 is the middle income family, the total amount of financial asset starts to affect the level of household income and it is positive influence, but there is deficient in the aspect of structure which shows that the low and the middle income families have encountered difficulties in the allocation aspect of financial asset structure and they have less understanding on financial information; Model 10 is the high income families, the impact of the total amount of financial asset disappears, the impact of allocation structure increases and the positive impact of investment-oriented financial product increases. This shows that the capacity of using financial tools of the high income family is stronger, but the capacity of low income family is weak and the financial asset allocation will increase the income gap.

\section{References}

[1] Gong Gang and Yangguang, Seeing the inequity of income distribution in China from functional income,J. Social Science in China, 2010(3):55-69.

[2] Zhu Ziyun, Multidimensional Empirical Analysis on Reasonability of Income Gap of Resident between provinces,J. Quantitative \& Technical Economics, 2016(2):22-41.

[3] Tian Qinggang, etc..Research on the impact of financial Value Development of Rural Families on Economic Behaviors of Peasant,J. Management World, 2015(10):180-181.

[4] Wang Shuchun and Wang Jun, Right Barrier, Polarization Effect and Regional Income Gap within An Integrated Region, J. Journal of Tianjin University of Commerce, 2015(4):11-16.

[5] Gan Li, etc. Analysis on Condition of Household assets in China and Housing Demand,J. Economic Research, 2013(4):1-14.

[6] Clarke George R. G., XuLixin Colin and Hengfu Zou. Finance and Income Inequality: What do the Data Tell US,J. Southern Economic Journal, 2006,72(3):578-596. 